\title{
Funcionalidade familiar e estratégias de enfrentamento em pessoas com lesão medular
}

\author{
Family functionality and coping strategies in people with spinal cord injury \\ Funcionalidad familiar y estrategias de afrontamiento en personas con lesión medular
}

Mayara Araújo Rocha' ${ }^{1}$ h htps://orcid.org/0000-0002-4991-0430

Alexsandro Silva Coura ${ }^{1}$ in https://orcid.org/0000-0002-0628-648X

Inacia Sátiro Xavier de França ${ }^{1}$ id https://orcid.org/0000-0002-2695-510X

Alexsandra Rodrigues Feijãa $0^{2}$ î https://orcid.org/0000-0002-8686-9502

Isabella Joyce Silva de Almeida ${ }^{1}$ ie nttps://orcid.org/0000-0001-8360-5897

Jamilly da Silva Aragão ${ }^{1}$ io https://orcid.org/0000-0002-8140-3180

Como citar:

Rocha MA, Coura AS, França IS, Feijão AR, Almeida IJ, Aragão JS. Funcionalidade familiar e estratégias de enfrentamento em pessoas com lesão medular. Acta Paul Enferm. 2021;34:APE000635.

DOI

http://dx.doi.org/10.37689/actaape/2021A0000635

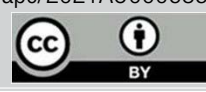

Descritores

Relações familiares; Adaptação psicológica; Traumatismos da medula espinal; Pessoas com deficiência

Keywords

Family relations; Adaptation, psychological; Spinal cord injuries; Disabled persons

Descriptores

Relaciones familiares; Adaptación psicológica; Traumatismos de la médula espinal; Personas con

discapacidad

Submetido

28 de Março de 2020

Aceito

9 de Dezembro de 2020

\section{Autor correspondente}

Mayara Araújo Rocha E-mail: mayararj83@gmail.com

\section{Resumo}

Objetivo: Analisar a funcionalidade familiar face as estratégias de enfrentamento de pessoas com lesão medular.

Métodos: Estudo transversal, desenvolvido em todas as 84 UBS de Campina Grande-PB, Brasil, no ano 2019. A população foi composta por todas as 54 pessoas com lesão medular adscritas nas unidades. Foi aplicado um questionário sociodemográfico e clínico, a Escala de Modos de Enfrentamento (EMEP) e o Apgar de Família. Realizou-se a regressão logística para estimar o odds ratio (OR).

Resultados: Verificou-se que a maioria é composta por homens, acima de 40 anos, não brancos, com credo religioso, escolaridade até o ensino fundamental, sem companheiro, com prole, renda familiar entre 1-2 salários mínimos, paraplégicos, com o tempo de lesão entre 2-15 anos, nível de comprometimento neurológico completo e de causa traumática. Quanto as estratégias de enfrentamento, as mais utilizadas foram a de religiosidade/pensamento fantasioso $(M=4,09)$ e com foco no problema $(M=3,93)$. As famílias eram funcionais em $72 \%$ dos casos. 0 modelo logístico demonstrou relações de probabilidade do foco na emoção $(0 R=0,539)$ (fator 2) e suporte social $(0 R=1,997)$ (fator 4) com a funcionalidade familiar.

Conclusão: Quanto maior a utilização de estratégias focadas na emoção e no problema menor é a funcionalidade familiar, e quanto maior o uso de estratégias de suporte social maior é a chance de ter uma boa funcionalidade.

\section{Abstract}

Objective: To analyze family functionality in view of coping strategies for people with spinal cord injury.

Methods: Cross-sectional study, developed in all 84 BHC in Campina Grande-PB, Brazil, in 2019. The population was composed of all 54 people with spinal cord injury registered in the Centers. A sociodemographic and clinical questionnaire, the Coping Modes Scale (CMS) and the Family Apgar were applied. Logistic regression was performed to estimate the odds ratio (OR).

Results: It was found that most of them were men, over 40 years old, non-white, with a religion, education up to elementary school, without a partner, with children, family income between 1-2 minimum wages, paraplegics, with injury time between 2-15 years, level of complete neurological impairment and traumatic cause. As for coping strategies, the most used were religiosity/fantasy thinking $(M=4.09)$ and focused on the problem $(\mathrm{M}=3.93)$. Families were functional in $72 \%$ of cases. The logistic model showed probability relationships of focus on emotion $(\mathrm{OR}=0.539)$ (factor 2$)$ and social support $(\mathrm{OR}=1.997)$ (factor 4) with family functionality. 
Conclusion: The greater the use of strategies focused on emotion and the problem, the lower the family functionality, and the greater the use of social support strategies, the greater the chance of having good functionality.

\section{Resumen}

Objetivo: Analizar la funcionalidad familiar frente a las estrategias de afrontamiento en personas con lesión medular.

Métodos: Estudio transversal, realizado en las 84 Unidades Básicas de Salud de Campina Grande, estado de Paraíba, Brasil, en el año 2019. La población estuvo compuesta por las 54 personas con lesión medular registradas en las unidades. Se aplicó un cuestionario sociodemográfico y clínico, la Escala de Modos de Afrontamiento y el Apgar de Familia. Se realizó la regresión logística para estimar la odds ratio (OR).

Resultados: Se verificó que la mayoría está compuesta por hombres, de más de 40 años, no blancos, con creencias religiosas, escolaridad hasta la educación primaria, sin compañero, con hijos, ingresos familiares entre 1 y 2 salarios mínimos, parapléjicos, con tiempo de lesión entre 2 y 15 años, nivel de compromiso neurológico completo y de causa traumática. Respecto a las estrategias de afrontamiento, las más utilizadas fueron la religiosidad/pensamiento fantasioso $(M=4,09)$ y el foco en el problema $(M=3,93)$. Las familias eran funcionales en el $72 \%$ de los casos. El modelo logístico demostró relaciones de probabilidad del foco en la emoción $(\mathrm{OR}=0,539)$ (factor 2) y el apoyo social $(\mathrm{OR}=1,997)$ (factor 4) con la funcionalidad familiar.

Conclusión: Cuanto más se utilizan estrategias centradas en la emoción y en el problema, menor es la funcionalidad familiar. Cuanto más se usan estrategias de apoyo social, mayor es la probabilidad de tener una buena funcionalidad.

\section{Introdução}

A Lesão Medular (LM) trata-se de uma condição que causa morte neural, inflamação e formação de cicatriz na medula, resultando em interrupção da função nervosa. ${ }^{(1)}$ Tal afecção está associada a diversas consequências de cunho econômico, social e psicológico. ${ }^{(2)}$

Acredita-se que a incidência da LM é de aproximadamente 10 casos por 100.000 pessoas no mundo, o que resulta em um quantitativo superior a 700.000 novos casos por ano. Quanto a prevalência, não se sabe ao certo devido à falta de notificação dessa condição. ${ }^{(2,3)}$ No Brasil, estima-se que a incidência seja de 16 a 26 casos novos por milhão de pessoas. ${ }^{(4)}$

Diante dos empasses vividos pós-lesão, é comum que se desencadeie um processo de ajuste psicológico que pode durar toda a vida. O comprometimento da capacidade do indivíduo realizar algumas atividades, torna a LM um fator estressante, sendo fundamental o uso de estratégias de enfrentamento para de diminuir o estresse e causar bem-estar psicológico. ${ }^{(5)}$

As estratégias de enfrentamento (coping) são esforços de cunho cognitivo e comportamental para lidar com o evento/causa estressora. ${ }^{(6)}$ As estratégias podem se apresentar em quatro principais formas: com foco no problema, com foco na emoção, pensamento fantasioso/religioso e suporte social. As estratégias com foco no problema procuram afetar diretamente o agente causador do estresse. Já aquelas com foco na emoção, tratam-se de estratégias utilizadas para lidar com a emoção causada pelo estresse..$^{(6,7)}$
Nesse contexto, é fundamental um bom funcionamento familiar, pois, aspectos como a adaptação, compartilhamento decisório e o tempo em que se passa junto aos familiares são importantes para o enfrentamento das condiçóes que a LM impóe. ${ }^{(8)}$ Portanto, quanto maior o suporte familiar que o indivíduo com LM possuir, melhor será o ajuste emocional e o uso das estratégias de enfrentamento. ${ }^{(9)}$

Nesse sentido, a Estratégia Saúde da Família (ESF) é de grande importância, pois a definição de família e como a mesma funciona, remete a uma melhor compreensão das práticas que devem rodear neste contexto. Logo, com aproximação dos profissionais da ESF podem ser percebidas condiçôes como sofrimentos, processos de ansiedade até mesmo de um mal enfrentamento de alguma condição, que outrora não foram observados. ${ }^{(10)}$

Nessa perspectiva, a investigação da funcionalidade familiar e estratégias de enfrentamento é imprescindível para uma melhor assistência de enfermagem a essa população, bem como a completude de ações que também envolvam não só o indivíduo afetado, mas também a sua família. Assim, acredita-se que tais aspectos contribuirão para melhor condição de vida, que envolvem um bem-estar psicológico e familiar.

Diante da justificativa, o presente estudo objetivou analisar associações entre a funcionalidade familiar e estratégias de enfrentamento de pessoas com LM. Dessa forma, a questão que emerge do estudo é: Qual a influência da funcionalidade familiar face ao uso das estratégias de enfrentamento de pessoas com LM? 


\section{Métodos}

Trata-se de um estudo de corte transversal, analítico, com uma abordagem quantitativa, desenvolvido no âmbito de todas as Unidades Básicas de Saúde (UBS) do município de Campina Grande, Paraíba, Brasil, no ano de 2019.

A população do estudo foi composta por todas as pessoas com LM que estavam adscritas em alguma das 84 Unidades Básicas de Saúde (UBS) do município. Portanto, 54 respondentes fizeram parte do estudo por atenderem aos critérios de inclusão: possuir 18 anos ou mais e apresentar um quadro de LM (completa ou incompleta). Já o critério excludente foi possuir alguma plegia que não fosse decorrente da LM.

A aproximação e identificação dos sujeitos deram-se por meio da visita domiciliar juntamente com o Agente Comunitário de Saúde (ACS) que por conhecerem os indivíduos que seriam o foco da pesquisa, levaram os pesquisadores junto aos domicílios. Tal processo foi realizado em dois momentos: o primeiro deu-se por meio da formação de vínculo e explicação do que se tratava a pesquisa e, caso o indivíduo elegível concordasse em participar, fora agendado um segundo momento para aplicação dos instrumentos I, II e III.

$\mathrm{O}$ instrumento I foi destinado a investigação de variáveis sociodemográficas. O II, denominado Escala de Modos de Enfrentamento de Problemas (EMEP), validado, ${ }^{(1)}$ trata-se de um questionário do tipo Likert de 1: "você nunca faz isso", a 5: "você faz isso sempre", composto por 45 itens relacionando algumas atitudes e pensamentos (estratégias) frente a um evento estressor, que na presente pesquisa é a LM. A EMEP realiza a identificação de quatro principais estratégias de enfrentamento ou fatores, sendo: estratégias de enfrentamento focadas no problema (Fator1); focadas na emoção (Fator2); práticas religiosas/pensamentos fantasiosos (Fator3) e a busca de suporte social (Fator4). O instrumento III foi o instrumento destinado a investigar a Funcionalidade Familiar: APGAR de Família, validado, ${ }^{(12)}$ que tem por objetivo avaliar o funcionamento familiar ao questionar acerca da percepçáo do indivíduo sobre sua família. $\mathrm{O}$ escore global pode demonstrar Elevada Disfunção Familiar (um a oito pontos); Moderada Disfunção Familiar (nove a 12 pontos) e Boa Funcionalidade Familiar (13 a 20 pontos).

Os dados coletados foram armazenados e analisados no programa STATA 14.0. Após a análise descritiva, foi realizado o teste de confiabilidade Alfa de Cronbach, que verifica a consistência interna do instrumento diante da aplicaçáo na amostra, sendo considerado o parâmetro de alfa $>0,8$ para uma boa confiabilidade. No que diz respeito a consistência interna dos instrumentos aplicados, o Apgar de Família e a EMEP foram analisados por meio do alfa de Cronbach, verificando-se uma boa confiabilidade de acordo com o alfa total dos instrumentos de 0,869 e 0,853 , respectivamente.

Foi utilizada a regressão logística binária, que consiste em uma regressão múltipla que relaciona uma variável categórica dicotômica, que na presente pesquisa foi a Funcionalidade Familiar (boa funcionalidade $=1$, disfuncional $=0$ ), e variáveis contínuas ou categóricas, sendo relacionadas as que correspondem aos fatores das estratégias de enfrentamento (Fator1, Fator2, Fator3 e Fator4). Para efeito de melhor ajuste do modelo foi realizada a clusterização utilizando a causa de lesão (traumática=1, e não traumática $=0$ ), sendo as variáveis de controle escolhidas por teoricamente afetarem algum dos aspectos estudados. Logo, tal técnica analisou a probabilidade de o evento ocorrer, por meio dos coeficientes de cada variável independente, sendo as medidas de odds ratio (OR) e p-valor $<0,05$. Foi realizado o procedimento de stepwise para definição dos modelos finais, como também, o teste de ajuste de Hosmer-Lemeshow.

Foram respeitados todos os preceitos éticos conforme preconiza a resolução 466/2012 do Conselho Nacional de Saúde (CNS) e o projeto foi aprovado no Comitê de Ética em Pesquisa da UEPB com o CAAE no 09335319.3.0000.5187 e parecer de no 3.225.266.

\section{Resultados}

A tabela 1 compila os dados do perfil sociodemográfico e clínico de pessoas com LM. Os achados 
demonstram a predominância de: homem $(68,5 \%)$, acima de 40 anos (53,7\%), com média de 43,9, não branco $(62,9 \%)$; com credo religioso $(75,9 \%)$; escolaridade até o ensino fundamental $(62,9 \%)$; sem companheiro $(61,1 \%)$; com prole $(66,7 \%)$; e com renda familiar entre 1-2 salários mínimos $(77,8 \%)$, considerando $\mathrm{R} \$ 998,00$ como valor da época da coleta. Quanto ao perfil clínico, a maioria são paraplégicos, com o tempo de lesão entre 2-15 anos, com média de 12,74, nível de comprometimento neurológico completo e de causa traumática.

Tabela 1. Perfil sociodemográfico e clínico de pessoas com LM $(\mathrm{n}=54)$

\begin{tabular}{lc}
\hline Variáveis & $\mathbf{n}(\%)$ \\
\hline Sexo & \\
Masculino & $37(68,5)$ \\
Feminino & $17(31,5)$ \\
Idade & \\
18-30 & $11(20,4)$ \\
$31-40$ & $14(25,9)$ \\
$41-50$ & $11(20,4)$ \\
>50 & $18(33,3)$ \\
Raça & \\
Parda & $26(48,1)$ \\
Branca & $20(37,1)$ \\
Preta & $8(14,8)$ \\
Crença & \\
Evangélica & $22(40,7)$ \\
Católica & $19(35,2)$ \\
Sem credo & $13(24,1)$ \\
Escolaridade & \\
Sem escolaridade & $3(5,6)$ \\
Alfabetização & $3(5,6)$ \\
Ensino fundamental & $28(51,6)$ \\
Ensino médio & $12(22,3)$ \\
Ensino superior & $8(14,9)$ \\
Estado civil & \\
Solteiro & $27(50,0)$ \\
Casado & $19(35,2)$ \\
Viúvo & $2(3,7)$ \\
Divorciado & $4(7,4)$ \\
União estável & $2(3,7)$ \\
Prole & \\
Com filhos & $36(66,7)$ \\
Sem filhos & $18(33,3)$ \\
Renda Familiar & \\
1-2 salários mínimos & $42(77,8)$ \\
>2 salários mínimos & $12(22,2)$ \\
Tempo de Lesão (anos) & \\
\hline$<2$ & $4(7,0)$ \\
2-15 & $35(65,0)$ \\
16- 48 & $15(28,0)$ \\
Tipo de plegia & \\
Tetraplegia & $12(22,2)$ \\
Paraplegia & $42(77,8)$ \\
Tipo de lesão & \\
Completa & $29(53,7)$ \\
Incompleta & $25(46,3)$ \\
Causa da Lesão & $31(57,4)$ \\
Traumática & $23(42,6)$ \\
Não-traumática & \\
\hline & \\
\hline &
\end{tabular}

n-frequência absoluta; \% - frequência relativa
Quanto as principais estratégias de enfrentamento utilizadas por pessoas com LM, a tabela 2 demonstra o uso da Religiosidade/pensamento fantasioso $(M=4,09)$, seguido da Focalizado na resolução de problemas $(\mathrm{M}=3,98)$ como os fatores principais.

Tabela 2. Medidas de tendência central e dispersão das estratégias de enfrentamento de pessoas com LM

\begin{tabular}{lccc}
\hline Estratégias de Enfrentamento (Fatores) & M & DP & Md \\
\hline Focalizado no problema & 3,98 & 1,688 & 5 \\
Focalizado na emoção & 1,33 & 1,082 & 1 \\
Religiosidade/pensamento fantasioso & 4,09 & 1,581 & 5 \\
Suporte social & 2,96 & 1,812 & 1 \\
\hline
\end{tabular}

M- Média; DP- Desvio Padrão; Md- Moda

Conforme apresentado na figura 1, o radar da funcionalidade familiar (Apgar de família) indica que $72,2 \%(n=39)$ dos participantes afirmam que suas famílias possuem boa funcionalidade familiar e, $27,8 \%(n=15)$, possuem alguma disfunção.

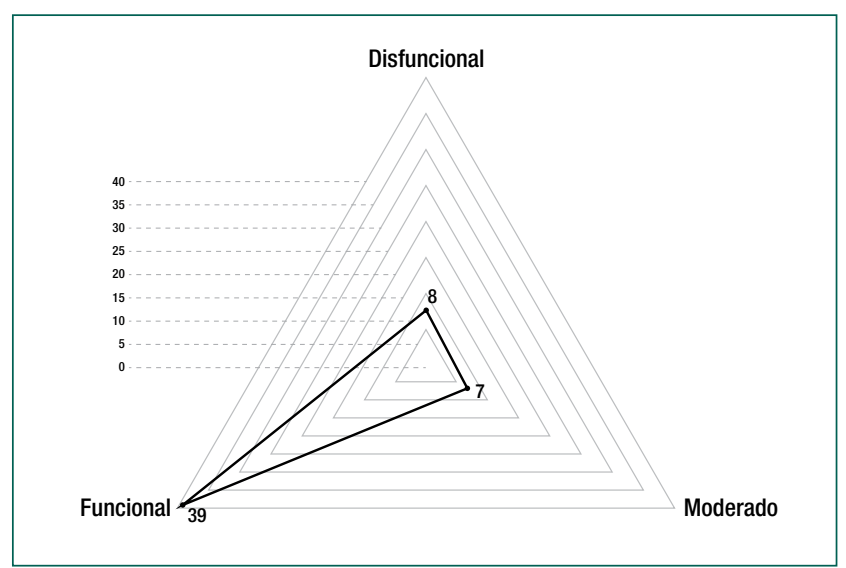

Figura 1. Radar da funcionalidade familiar (Apgar de Família) de pessoas com LM.

A relação entre a funcionalidade familiar e estratégias de enfrentamento e aspectos clínicos está indicada na tabela $3 \mathrm{com}$ a análise de regressão logística. $\mathrm{O}$ modelo 1 (mais completo) apresentou significância entre todos os fatores e variáveis de controle, exceto o fator 3 e o estado civil divorciado. No fator 1 , focado no problema (OR 0,651; IC 0,490-0,865), apresentou-se relação com a Funcionalidade Familiar, de maneira que a utilização de tal estratégia reduz as chances de possuir uma boa funcionalidade familiar. 
Tabela 3. Modelos de regressão logística ajustados entre a funcionalidade familiar e fatores das estratégias de enfrentamento e variáveis de controle

\begin{tabular}{|c|c|c|c|c|c|c|c|c|c|}
\hline Variáveis & $\begin{array}{c}\text { (1) } \\
\text { Funcionalidade } \\
\text { familiar }\end{array}$ & IC (95\%) & $p$-value & $\begin{array}{c}\text { (2) } \\
\text { Funcionalidade } \\
\text { familiar }\end{array}$ & IC (95\%) & $p$-value & $\begin{array}{c}\text { (3) } \\
\text { Funcionalidade } \\
\text { familiar }\end{array}$ & IC (95\%) & $p$-value \\
\hline Foco no problema & $0,651^{\star \star \star}$ & $(0,490-0,865)$ & 0,003 & & & & & & \\
\hline Foco na emoção & $0,539^{\star \star}$ & $(0,319-0,910)$ & 0,021 & $0,658^{\star \star *}$ & $(0,532-0,813)$ & 0,000 & $0,657^{\star \star \star}$ & $(0,530-0,815)$ & 0,000 \\
\hline Suporte social & $1,997^{\star \star \star}$ & $(1,467-2,720)$ & 0,000 & & & & $1,297^{\star \star \star}$ & $(1,104-1,523)$ & 0,002 \\
\hline Tempo de lesão & $0,932^{\star \star \star}$ & $(0,892-0,975)$ & 0,002 & $0,952^{\star \star \star}$ & $(0,919-0,986)$ & 0,007 & $0,966^{\star \star \star}$ & $(0,954-0,978)$ & 0,000 \\
\hline Católico & $21,10^{\star \star}$ & $(1,710-260,4)$ & 0,017 & & & & & & \\
\hline Evangélico & $2,391^{\star \star}$ & $(1,061-5,390)$ & 0,036 & & & & & & \\
\hline Solteiro & $111,1^{\star \star \star}$ & $(37,31-330,5)$ & 0,000 & & & & & & \\
\hline Casado & $13,67^{\star \star \star}$ & $(11,08-16,85)$ & 0,000 & & & & & & \\
\hline Divorciado & & & & $0,141^{\star *}$ & $(0,020-0,994)$ & 0,049 & $0,126^{\star \star \star}$ & $(0,049-0,322)$ & 0,000 \\
\hline Quantidade de filhos & $2,552^{\star}$ & $(0,983-6,631)$ & 0,054 & $1,698^{\star \star \star}$ & $(1,227-2,352)$ & 0,001 & $1,580^{\star \star}$ & $(1,089-2,291)$ & 0,016 \\
\hline Constante & $2,197^{\star \star \star}$ & $(1,836-2,630)$ & 0,000 & $60,72^{\star \star \star}$ & $(15,88-232,1)$ & 0,000 & $37,44^{\star \star}$ & $(1,185-1,183)$ & 0,040 \\
\hline Hosmer-Lemeshow chi2(8) (10group) & 3,39 & & & 8,65 & & & 15,76 & & \\
\hline Prob $>$ chi2 & 0,9079 & & & 0,3724 & & & 0,0459 & & \\
\hline Pseudo-R2 & 0,343 & & & 0,176 & & & 0,164 & & \\
\hline Observações & 54 & & & 54 & & & 54 & & \\
\hline
\end{tabular}

${ }^{\star \star \star} p<0,01,{ }^{\star \star} p<0,05,{ }^{\star} p<0,1 ;$ IC- intervalo de confiança; chi2- teste de qui-quadrado

Ainda no modelo 1 , no que concerne ao fator 2, focado na emoção (OR 0,539; IC 0,319-0,910), a relação demonstrou que quanto maior a utilização de tais estratégias, menores as chances de possuir uma boa funcionalidade familiar. Já no fator 4 , de suporte social (OR 1,997; IC 1,467-2,720) a regressão demonstrou que quanto mais se utiliza tal estratégia, aumenta em aproximadamente duas vezes as chances de possuir uma boa funcionalidade familiar.

Tratando-se das principais variáveis de controle, observou-se que ser católico (Relig2) (OR 21,10; IC 1,710-260,4) aumenta as chances em 21 vezes de possuir uma boa funcionalidade familiar, se sobrepondo a não possuir religião ou ser evangélico. Quanto ao estado civil, observa-se que ser solteiro (OR 111,1) aumenta a probabilidade de ter boa funcionalidade em 100 vezes, comparado aos outros estados civis. E por fim, acredita-se que possuir filhos (OR 2,552) aumenta a probabilidade de ter uma boa funcionalidade familiar.

Nos modelos 2 e 3 foram suprimidas as variáveis "Solteiro" e "Casado" (respectivamente) por apresentarem Odds Ratio muito diferente das demais variáveis. Após o procedimento de stepwise, tais modelos suprimiram as variáveis Fator1 (modelo 2 e 3) e Fator4 (modelo2). Portanto, as variáveis "solteiro" e "casado", apesar de gerar um resultado bem diferente, são importantes para contribuir na explicação do modelo.
Além disso, o pseudo R2 do modelo 1 (pseudo $\mathrm{R} 2=0,34$ ) foi maior do que o modelo 2 (pseudo $\mathrm{R} 2=0,17$ ) e 3 (pseudo $\mathrm{R} 2=0,16$ ). Sendo assim, o modelo1 é considerado o mais ajustado e com melhor poder de explicação.

\section{Discussão}

No tocante ao perfil sociodemográfico, a maioria ser do sexo masculino e de baixa escolaridade coaduna com um estudo internacional, cujo objetivo central foi descrever a epidemiologia dos pacientes com LM em tratamento, no qual mais de $80 \%$ dos indivíduos eram homens. Tal ocorrência se explica pelo fato de que estão mais susceptíveis, pelo próprio contexto social que se inserem, a sofrerem lesóes traumáticas, relacionadas a armas de fogo, acidentes de trabalho e automobilísticos. ${ }^{(13)}$

No que concerne ao nível de escolaridade, acredita-se que muitos por possuírem debilidade física, o que pode causar exposição social, não tenham concluído os estudos pós-lesão, por isso, a predominância do ensino fundamental. ${ }^{(13)}$ Ademais, há uma associação entre a baixa escolaridade e a prontidão para o autocuidado, o que favorece a exposiçóes que possuem potencial elevado de riscos à saúde.

Quanto a faixa etária, sendo maior que 50 anos, um estudo brasileiro desenvolvido com homens pes- 
cadores com LM, apresentou resultado semelhante, com uma média de 50,2 anos. $^{(14)} \mathrm{O}$ que contrapóe a maioria dos estudos na área, que destacam a prevalência de LM em adultos jovens. ${ }^{(13,15,16)}$ Tal discrepância explica-se pela diferença no recrutamento amostral, pois os estudos tratam a LM decorrente de causas traumáticas, que são mais prevalentes na faixa etária adulto-jovem, enquanto o presente estudo considerou causas traumáticas e não traumáticas. ${ }^{(15)}$

No que concerne as características clínicas relacionadas a LM, os indivíduos da presente pesquisa apresentaram paraplegia, decorrente de trauma, com uma diferença pouco significativa relacionada ao tipo de comprometimento da lesão. Nesse sentido, dois estudos ${ }^{(17,18)}$ desenvolvidos na Suécia e China, demonstraram as mesmas condiçóes clínicas, sendo paraplegia, em 76\% e 61\% dos casos respectivamente, de origem traumática e de forma completa/incompleta, nessas últimas não havendo diferenças significantes. Nesse sentido, as causas traumáticas merecem atenção, pois evidencia-se que a maioria dos casos contribuem para ocorrência de complicaçóes associadas na fase aguda e crônica. ${ }^{(19)}$

Tratando-se das estratégias de enfrentamento, em sua maioria o grupo de estratégias focadas no problema e na religiosidade/pensamento fantasioso, foram as mais utilizadas. A frequência de estratégias de enfoque no problema corrobora outro estudo desenvolvido na Colômbia com a mesma população. (20) Assim, as estratégias focadas no problema sugerem forças para enfrentar a causa estressora, que no presente caso é a LM, estando assim, associadas a menor taxa de depressão e maior ajuste social. ${ }^{(21,22)}$

A utilização das estratégias de enfrentamento de cunho religioso/fantasioso está relacionada a possuir alguma crença. Nesse sentido, acredita-se que a espiritualidade interfira positivamente em aspectos físicos e mentais, sobretudo em pessoas com condiçôes crônicas, tal como a LM, o que resulta em um melhor enfrentamento da situação que lhe é imposta, melhorando aspectos da Qualidade de Vida (QV) e reduzindo a ocorrência de depressão e ansiedade, tal como conclui um estudo desenvolvido no Irã acerca do enfrentamento religioso. ${ }^{(23,29)}$

A prática religiosa é uma estratégia facilitadora no enfrentamento do cotidiano do cuidado e poten- te maneira de socialização. A perpetuação de uma condição limitante, conclama artifícios que aliviem essa tensão contínua. Considerando que para a aproximação de um bem-estar pleno, que é a junção da saúde biopsicossocial, os aspectos religiosos beneficiam os aspectos psicológicos que interagem diretamente na saúde física e social. ${ }^{(24,30)}$ Dessa forma, entende-se que os enfermeiros devem considerar essas estratégias em suas práticas de cuidados.

Outrossim, no que concerne o funcionamento familiar, foi considerado bom pela maioria das famílias, o que corrobora um estudo brasileiro cujo objetivo principal foi identificar a coesão, adaptação, flexibilidade e funcionalidade familiar de pessoas com LM, o qual demonstrou que em $61,4 \%$ dos eventos, apresentaram boa funcionalidade familiar. Porém, ainda existe um considerável quantitativo de disfunção familiar, seja ela moderada ou grave, que merece destaque, pois acredita-se que a construção familiar ajuda o indivíduo a passar por diversos obstáculos no processo de adaptação, visto que tal momento ocorre em conjunto. ${ }^{(9,24)}$

O compartilhamento deste universo com a rede de apoio que a família forma desvela um refúgio a pessoa com LM, constituindo um ambiente que alberga zelo e solicitude, transcendendo-os do peso da condição crônica a um universo de acolhimento e partilha, legitimando os vínculos afetivos familiares. ${ }^{(31)}$

Logo, visando uma abordagem humanística e efetiva por parte dos enfermeiros e demais profissionais de saúde, é imprescindível que eles atentem para a funcionalidade familiar durante o processo de reabilitação, visto que há dificuldades, sobretudo, no processo adaptativo a essa condição crônica, tanto para pessoa com LM quanto para a família. ${ }^{(25)}$

Quanto aos dados inferenciais, a regressão logística proposta demonstrou que a utilização dos fatores de enfoque no problema reduz as chances de ter uma boa funcionalidade familiar. Nesse sentido, o enfoque no problema está relacionado a esforços para solucionar o fator que lhe causa estresse, pois a presença da condição crônica no interior familiar pode interferir na estrutura da mesma, visto que tal adaptação funciona em conjunto. ${ }^{(24)}$

Outra questão importante é o aparecimento de estresse pós-trauma, no qual as relaçóes familiares 
podem estar fomentando funçóes protecionistas ou até mesmo agravando a condição, diante de uma família disfuncional. ${ }^{(32)}$ Nesse contexto, o indivíduo pode parecer decisivo em mudar a situação, mas, em algumas vezes com uma família disfuncional. Um estudo brasileiro sobre a funcionalidade familiar em pessoas com LM demonstrou que, apesar da boa funcionalidade, existe uma insatisfação com a família, sobretudo na comunicação entre pares. ${ }^{(9)}$

Outra investigação de método semelhante realizada no México, junto a pacientes com deficiência permanente, demonstrou que a maioria da amostra obteve boa funcionalidade $(93,2 \%)$, e essas pessoas utilizaram estratégias com foco no estresse (problema), o que contrapóe o resultado aqui apresentado. (26) Tal contraposição pode estar relacionada com as diferenças culturais e familiares entre os países, bem como com as distintas peculiaridades inerentes aos níveis de lesão das amostras consideradas para os dois estudos.

Quanto ao fator 2 com enfoque na emoção, acredita-se que a utilização de tais estratégias diminua as chances de possuir uma boa funcionalidade familiar. $\mathrm{O}$ enfoque na emoção é inteiramente preenchido com uma elevada carga emocional, o que muitas vezes pode gerar mecanismos de distanciamento, bem como expressão de sentimentos profundos de culpa e de culpar os outros, gerando conflitos familiares e desconstrução da boa funcionalidade. ${ }^{(11,27)}$

Já o fator 4, de suporte social demonstrou que quanto mais se utiliza tal estratégia, aumenta em aproximadamente duas vezes as chances de possuir uma boa funcionalidade familiar. Nesse sentido, a busca por suporte social, seja ele de amigos ou familiares, se configura como uma estratégia que visa aproximar os que estão ao redor para um melhor enfrentamento do problema. Um estudo brasileiro sobre a rede de apoio de pessoas com LM, identificou que o principal apoio vem da família, sendo ela responsável pelo cuidado e preparação para uma melhor adaptação psicológica do indivíduo. ${ }^{26,28)}$

Por tudo, acredita-se que a utilização de estratégias de enfrentamento em busca de suporte social sejam fundamentais no funcionamento familiar desses indivíduos, sendo o contrário também ver- dadeiro, o que remete a eminente necessidade da utilização de mecanismos que envolvam os familiares no processo de reabilitação, sobretudo quando consideram-se os aspectos psicológicos.

Os resultados proporcionaram reflexóes para a práxis da enfermagem no âmbito da ESF, sobretudo na avaliaçáo dos dois aspectos aqui estudados. Uma das formas de trazer tais particularidades está na consulta de enfermagem e visita domiciliar aos indivíduos com LM, cumprindo preceitos da integralidade do cuidado a pessoas com deficiência.

Este trabalho apresenta algumas limitaçóes. Primeiro, acredita-se que apesar do estudo ser censitário, o tamanho populacional foi reduzido, portanto, recomenda-se que o mesmo seja replicado em uma abrangência maior. Segundo, o desenho do estudo não permite obter uma relação de causalidade por não possibilitar um acompanhamento da amostra no decorrer do tempo.

\section{Conclusão}

Verificaram-se relaçóes de probabilidade entre o foco no problema, na emoção e suporte social, sendo o último relacionado positivamente. Portanto, conclui-se que quanto maior a utilização de estratégias focadas na emoção e no problema menor é a funcionalidade familiar, e quanto maior o uso de estratégias de suporte social maior é a chance de ter uma boa funcionalidade. Logo, os enfermeiros e demais profissionais da saúde devem incluir os aspectos de suporte social em seus planos de cuidados, visto que fazem parte do processo de reabilitação e recuperação das pessoas com LM.

\section{Colaborações}

Rocha MA, Coura AS contribuíram na concepção do projeto, análise e interpretação dos dados e redação. Feijão AR, França ISX, Almeida IJS e Aragão JS contribuíram na revisão crítica relevante do conteúdo intelectual e aprovação da versão final a ser publicada. 


\section{Agradecimentos}

\section{A Fundação de Apoio à Pesquisa do Estado da Paraíba (FAPESQ-PB), pela bolsa de mestrado con- cedida a Rocha MA.}

\section{Referências}

1. He Z, Zang H, Zhu L, Huang K, Yi T, Zhang S, Cheng S. An antiinflammatory peptide and brain-derived neurotrophic factor-modified hyaluronan-methylcellulose hydrogel promotes nerve regeneration in rats with spinal cord injury. Int J Nanomedicine. 2019;18(14):721-32.

2. Teplitsky S, Murphy A, Shenot PJ. Knowledge gaps in urologic care of female spinal cord injury patients. Curr Urol Rep. 2019;20(5):21.

3. Kumar R, Lim J, Mekary RA, Rattani A, Dewan MC, Sharif SY, et al. Traumatic Spinal Injury: Global Epidemiology and Worldwide Volume. World Neurosurg. 2018;113:e345-63.

4. Ahuja CS, Martin AR, Fehlings MG. Fehlings, M. Recent advances in managing a spinal cord injurysecondary to trauma. F1000 Res. 2016;5:1017.

5. Cavalcante ES, Pessoa JJ, Freire IL, Faro A. C Mi e, Torres G V, Miranda F A N. Spinal cord injury due to diving accidents and stress among artisanal fishers. Texto Contexto Enferm. 2017;26(2):e00190016

6. Garrido S. The influence of personality and coping style on the affective outcomes of nostalgia: is nostalgia a healthy coping mechanism or rumination? Personal Indiv Differ. 2018;120:259-64.

7. Noda T, Takahashi Y, Murai T. Coping mediates the association between empathy and psychological distress among Japanese workers. Personal Individ Differ. 2018;124:178-83.

8. Vera I, Lucchese R, Nakatani AY, Pagotto V, Montefusco SR, Sadoyama G. Family functionality in oldest old household residents. Rev Bras Enferm. 2015;68(1):61-8.

9. Trierveiler KS, Ramos FR, Schoeller SD, Nogueira GC, Martins MM, Schneider DG. Funcionalidade familiar da pessoa com lesão medular. Texto Contexto Enferm. 2015;24(4):993-1002.

10. Silva $M C$, Silva $L$, Bousso RS. A abordagem à família na Estratégia Saúde da Família: uma revisão integrativa da literatura. Rev Esc Enferm USP. 2011;45(5):1250-5.

11. Seidl EM, Troccoli BT, Zannon CM. Análise fatorial de uma medida de estratégias de enfrentamento. Psicol Teor Pesqui. 2001;17(3):22534.

12. Duarte YA. Família: rede de suporte ou fator estressor- a ótica de idosos e cuidadores familiares [tese]. São Paulo: Escola de Enfermagem, Universidade de São Paulo; 2001. 196 p.

13. Lemos HF, Neves SM, Lima TM, Carvalho AF. Epidemiological profile of patients with spinal cord injury attended at a rehabilitation center. ReonFacema. 2017;3(3):557-60.

14. Cavalcante ES, Pessoa JM, Freire IL, Cavalcante CA, Miranda F A N. Representações sociais de pescadores com lesão medular: repercussões e trajetória de vida. Rev Bras Enferm. 2017;70(1):13945.
15. Corrêa LD, Lopes Neto D, Llapa Rodriguez EO. Qualidade de vida de pessoas com lesão medular traumática. Cogitare Enferm. 2015;20(4):695-700.

16. Carvalho ZM, Andrade LM, Pinheiro AK, Studar RM. influência da espiritualidade, religião e crenças na qualidade de vida de pessoas com lesão medular. Texto Contexto Enferm. 2015;24(3):792-800.

17. Divanoglou A, Augutis M, Sveinsson T, Hultling C, Levi R. Self-reported health problems and prioritized goals in community-dwelling individuals with spinal cord injury in Sweden. J Rehabil Med. 2018;50(10):872-8.

18. Wang $Y$, Zhao X, Xie H. Quality of life and its predictors in people with traumatic spinal cord injury in mainland China. Spinal Cord. 2019;57(9):739-46.

19. Eckert MJ, Martin MJ. Trauma: spinal cord injury. Surg Clin North Am. 2017;97(5):1031-1045.

20. Torregrosa-Ruiz M, Tomás-Miguel JM. Diferencias de sexo en el uso de estrategias de afrontamiento en personas con lesión medular. Aquichan. 2017;17(4):448-59.

21. Andrade Paiva Morero J, Rodrigues Bragagnollo G, Signorini Santos MT. Estratégias de enfrentamento: uma revisão sistemática sobre instrumentos de avaliação no contexto brasileiro. Rev Cuid (Bucaramanga). 2018;9(2):2257-68.

22. Pollock K, Dorstyn D, Butt L, Prentice S. Posttraumatic stress following spinal cord injury: a systematic review of risk and vulnerability factors. Spinal Cord. 2017;55(9):800-11.

23. Rahnama P, Javidan AN, Saberi H, Montazeri A, Tavakkoli S, Pakpour $\mathrm{AH}$, et al. Does religious coping and spirituality have a moderating role on depression and anxiety in patients with spinal cord injury? A study from Iran. Spinal Cord. 2015;53(12):870-4.

24. Wilson CS, Forchheimer M, Heinemann AW, Warren AM, McCullumsmith C. Assessment of the relationship of spiritual well-being to depression and quality of life for persons with spinal cord injury. Disabil Rehabil. 2017;39(5):491-6.

25. Machado WC. 0 papel do enfermeiro no cuidar de clientes portadores de deficiência. São Paulo: Entre Amigos - Rede de Informações sobre Deficiência; 2012.

26. Rosales-Cordova NC, Garrido-Perez S, MCarrillo-Ponte F. Funcionalidad familiar y afrontamiento en pacientes con incapacidad permanente en una Unidad Médica Familiar. Horiz Sanitario. 2017;16(2):127-37.

27. Costa L, Lordes RG, Fraga D, Santana NM, Bubach S, Leite FM. Estratégias de enfrentamento adotadas por mulheres vítimas de violência. Rev Enfer UFRJ (Rio de Janeiro). 2018; 26:e19334.

28. Ruiz AG, Barreto MS, Peruzzo HE, Schoeller SD, Descesario MN, Marcom SS. Atuação da rede de apoio a pessoas com lesão medular. REME Rev Mineira Enferm. 2018;16(112):e1116.

29. Rodrigues FF, Santos MA, Teixeira CR, Gonela JT, Zanetti ML. Relação entre conhecimento, atitude, escolaridade e tempo de doença em indivíduos com diabetes mellitus. Acta Paul Enferm. 2012;25(2):284-90.

30. Garanito MP, Cury MR. A espiritualidade na prática pediátrica. Rev Bioet. 2016;24(1):49-53.

31. Cruz DM, Nascimento LR, Vieira da Silva DM, Dornelles Schoeller S. Redes de apoio à pessoa com deficiência física. Cienc Enferm. 2015;21(1):23-33.

32. Souza CM, Vizzotto MM, Gomes MB. Relação entre violência familiar e transtorno de estresse pós-traumático. Psic Saúde Doenças. 2018;19(2):222-33. 